\title{
Retinal breaks due to intravitreal ocriplasmin
}

\author{
Ruwan A Silva \\ Darius M Moshfeghi \\ Theodore Leng \\ Byers Eye Institute at Stanford, \\ Stanford University School of \\ Medicine, Palo Alto, CA, USA
}

This article was published in the following Dove Press journal:

Clinical Ophthalmology

25 August 2014

Number of times this article has been viewed

\begin{abstract}
Ocriplasmin represents a new treatment option for numerous vitreoretinopathies involving an abnormal vitreomacular interface. While the drug may circumvent the traditional risks of surgical treatment, pharmacologic vitreolysis is not devoid of risk itself. This report presents two cases, one of vitreomacular traction syndrome and the other of a full-thickness macular hole, both of which were treated with an intravitreal injection of ocriplasmin. Notably, in both cases, vitreomacular traction of the macula appears to have been alleviated; however, failure to completely relieve vitreoretinal traction from the peripheral retina generated retinal breaks with one patient eventually developing a macula-involving retinal detachment. Thus, even in instances of 'successful' pharmacologic treatment of vitreomacular traction, continued follow-up evaluation is essential.
\end{abstract}

Keywords: posterior vitreous detachment, retinal detachment, vitreomacular traction, ocriplasmin, retinal break, macular hole, laser retinopexy

\section{Introduction}

The vitreoretinal interface underlies a significant proportion of retinal pathology that has previously required surgical intervention. The spectrum of these diseases, while previously limited to macular holes, myopic schisis-like retinopathy and vitreomacular traction syndrome, are now suspected to also encompass diabetic retinopathy, retinal vein occlusion, subtypes of cystoid macular edema and even exudative age-related macular degeneration..$^{1-3}$ The extracellular matrix comprising the vitreoretinal interface, composed mainly of collagen and the proteoglycans fibronectin and laminin, ${ }^{4}$ bridges the cortical vitreous to the retina's internal limiting membrane and has most recently been pharmacologically targeted by ocriplasmin (Jetrea ${ }^{\circledR}$; ThromboGenics, Inc., Iselin, NJ, USA) a truncated catalytic domain of plasmin. ${ }^{5}$

Following a series of Phase II and III clinical trials, the most pivotal of which were collectively called the Microplasmin for Intravitreous Injection - Traction Release without Surgical Treatment (MIVI-TRUST) study, ${ }^{6}$ the United States Food and Drug Administration (FDA) granted approval of ocriplasmin for symptomatic vitreomacular adhesion on October 18, 2012. While initial experiences with ocriplasmin have generally been favorable since its commercial availability in January of $2013,{ }^{7}$ surveillance of post-approval drug safety remains critical. Toward that end, we report two cases of retinal breaks occurring after intravitreal injection of ocriplasmin.

\section{Case I}

A 66-year-old female was referred to the vitreoretinal surgery clinic for decreased central visual acuity in the right eye. Her best corrected visual acuity (BCVA) was 20/70-1 and 20/20 in the right and left eyes, respectively. Anterior segment examination revealed $1+$ nuclear sclerotic cataracts bilaterally. Posterior segment examination of the right eye was notable for a blunted foveal light reflex and central macula thickening
Correspondence: Theodore Leng Wersity School of Medicine, 2452 Watson Ct, Palo Alto, CA 94303, USA Tel +l 6504984264

Fax + I 8885652640

Email tedleng@stanford.edu submit your manuscript | www.dovepress.com

Dovepress

http://dx.doi.org/10.2147/OPTH.S68037 
due to vitreomacular traction with no Weiss ring seen on examination. Peripheral retinal examination of the right eye revealed no retinal breaks. Examination of the left eye was unremarkable. Spectral-domain optical coherence tomography (SD-OCT) of the right eye demonstrated vitreomacular traction yielding foveal distortion as well as intraretinal and subretinal fluid (Figure 1). After obtaining informed consent, the patient was treated with $125 \mu \mathrm{g}$ in $100 \mu \mathrm{L}$ of intravitreal ocriplasmin, which was injected into the inferotemporal quadrant of the right eye. Routine follow-up 1 week later revealed symptomatic improvement in central vision of the right eye with examination demonstrating a stable BCVA of 20/70. Posterior segment examination demonstrated release of vitreomacular traction with subretinal fluid. SD-OCT confirmed posterior hyaloid separation from the central macula with improvement in intraretinal fluid and persistent subretinal fluid (Figure 2). The peripheral retinal exam was notable only for a nasal, flame shaped hemorrhage in the right eye with no retinal breaks noted. Twelve days after ocriplasmin administration, the patient developed acute onset of flashing lights in the right eye. Her BCVA was 20/60 with posterior examination now demonstrating a Weiss ring, continued subfoveal fluid and release of vitreomacular traction. However, peripheral examination revealed a superotemporal operculated retinal hole with a surrounding cuff of subretinal fluid. The patient was treated with laser retinopexy in the right eye without complication. Over the next 2 months the patient's BCVA improved to 20/40 though she noted intermittent, though self-limited, flashing lights in her right eye. Posterior segment examination at 2 months demonstrated residual subfoveal fluid, laser scars surrounding the superotemporal retinal break and no other retinal breaks. SD-OCT confirmed the continued presence of subfoveal fluid, though progressive improvement in the foveal contour of the right eye was noted (Figure 3). Visual acuity and examination remained stable at 6 months.

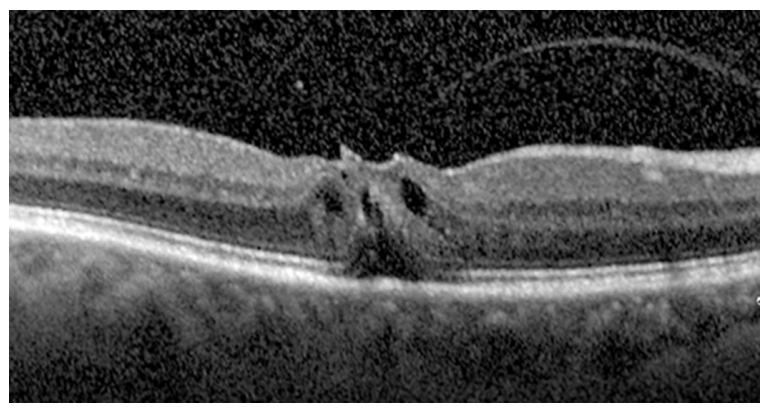

Figure I Pre-injection spectral-domain optical coherence tomography image of the right eye in Case I demonstrating vitreomacular traction syndrome. Note: The fovea appears distorted with intraretinal and subretinal fluid.

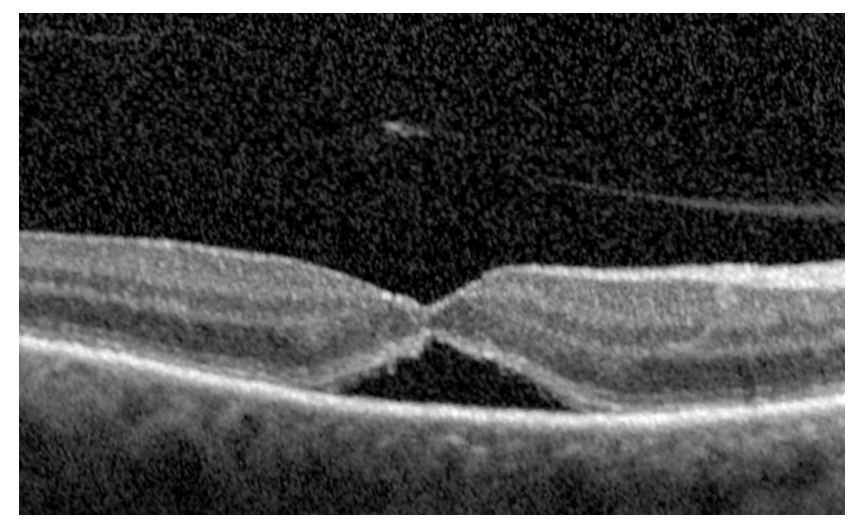

Figure 2 Spectral-domain optical coherence tomography image of the right eye in Case I, I week after ocriplasmin injection.

Notes: The prior vitreomacular traction and intraretinal fluid appear improved, though persistent subretinal fluid remains.

\section{Case 2}

A 65-year-old female was referred to the vitreoretinal surgery clinic for a decline in central visual acuity in the left eye. Her BCVA was 20/25-1 and 20/80-1 in the right and left eyes, respectively. Anterior segment examination revealed trace nuclear sclerotic cataracts bilaterally. Posterior segment examination of the right eye was unremarkable while examination of the left eye demonstrated a full-thickness macular hole with vitreomacular traction. Peripheral retinal examination of both eyes was unremarkable. SD-OCT (Figure 4) of the left eye demonstrated a full-thickness macular hole with vitreomacular traction. After obtaining informed consent, the patient was treated with $125 \mu \mathrm{g}$ in $100 \mu \mathrm{L}$ of intravitreal ocriplasmin which was injected into the inferotemporal quadrant of the left eye. At follow-up examination 1 week later, the patient revealed she had suffered a 4 day history of flashing lights in the left eye starting 1 day after ocriplasmin

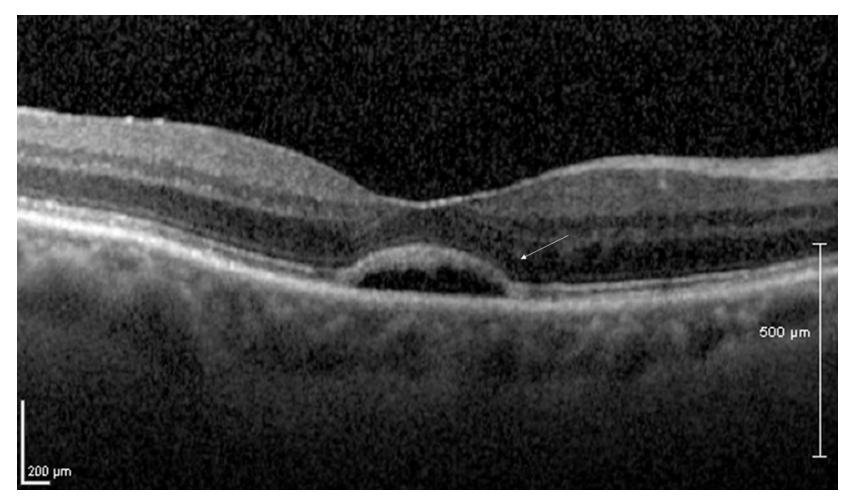

Figure 3 Spectral-domain optical coherence tomography image of the right eye in Case I, 8 weeks after ocriplasmin injection.

Notes: Though subretinal fluid persists, it is improved from the immediate postinjection appearance. Additionally, the outer neurosensory retinal appearance (external limiting membrane, inner segment/outer segment and cone outer segment tips) also appears improved from the post-injection appearance (arrow). 


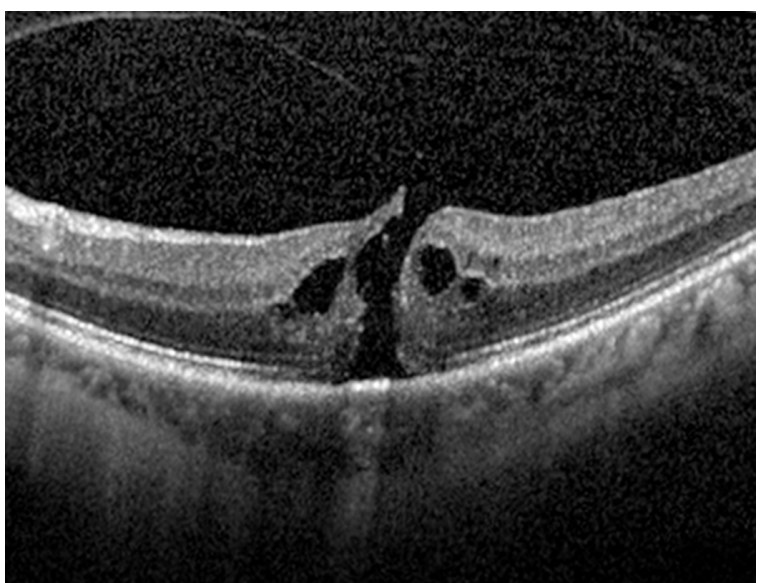

Figure 4 Pre-injection spectral-domain optical coherence tomography image of the left eye in Case 2 demonstrating a stage 3 macular hole and intraretinal cystic changes.

Note: Vitreomacular traction is seen overlying the central macula.

injection. Though this subsided, she then noted a shadow in the nasal and central regions of her left visual field which started 6 days after her ocriplasmin injection. Her BCVA was 20/25 (eccentric) in the left eye. Posterior segment examination demonstrated interval macular hole closure, but also revealed three horseshoe retinal tears with an associated macula-involving retinal detachment (Figure 5). The patient underwent immediate and successful retinal detachment repair surgery by scleral buckling, pars plana vitrectomy, air-fluid exchange, endolaser and $14 \% \mathrm{C}_{3} \mathrm{~F}_{8}$ gas-injection. At the 6 month follow-up visit, the patient was noted to have

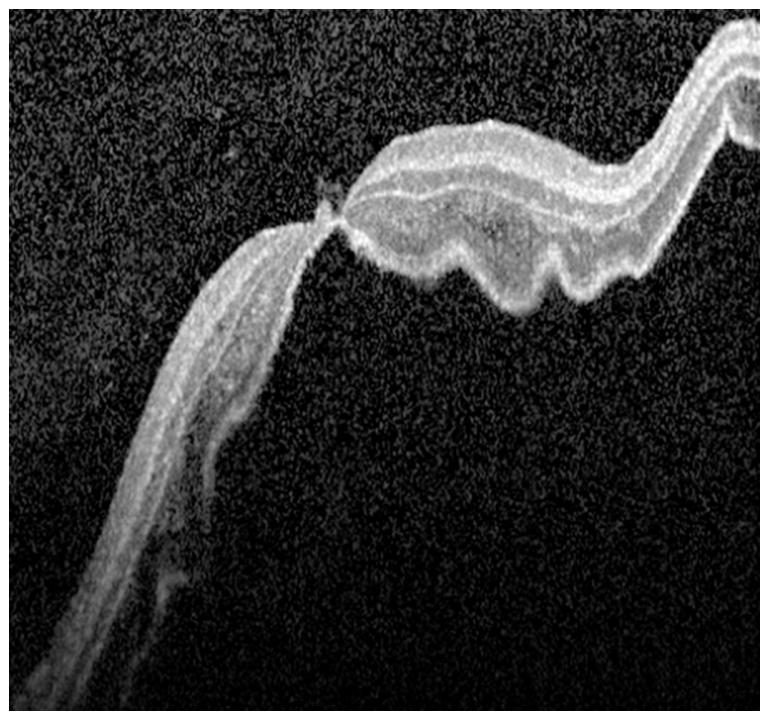

Figure 5 Spectral-domain optical coherence tomography image of the left eye in Case 2, I week after ocriplasmin injection.

Notes: The macular hole appears closed, though a macula-involving retinal detachment is now present. a best-corrected visual acuity of 20/50 with (visually significant) nuclear sclerotic and posterior subcapsular cataract. Posterior segment examination demonstrated the macula and peripheral retina to be attached with a good encircling buckle effect and without any additional retinal breaks.

\section{Discussion}

While ocriplasmin represents a potent new tool in the treatment of vitreomacular traction, careful monitoring of treated patients, as with any novel therapy, remains paramount in assessing both its efficacy and safety.

While the above represents the first case series of retinal breaks occurring after intravitreal ocriplasmin administration, their occurrence in and of themselves is not without precedent. The MIVI I trial (Phase I/II clinical trial), which enrolled 60 patients, reported a far peripheral retinal tear with an associated retinal detachment noted on post-injection day $1 .{ }^{8}$ A later Phase II trial, in which 92 patients received varying doses of ocriplasmin, also reported two retinal tears. These tears occurred within 7 days of intravitreal injections but the exact timing of their occurrence is unclear. ${ }^{9}$ Similarly, the MIVI-TRUST studies (Phase II/III clinical trials) reported two post-injection retinal tears with associated retinal detachments in the 464 patients treated with ocriplasmin though the exact timing of these tears with respect to the injections was again not reported. ${ }^{6}$ Given the timing of each of the prior reported instances, the breaks may have been a result of the intravitreal injection itself rather than the pharmacologic action of ocriplasmin. There is some evidence supporting this theory: the MIVI I trial authors reported that during surgical repair of the aforementioned retinal tear and detachment, the vitreous was found still firmly adherent to the retina. ${ }^{8}$

Regardless of the cause of previously reported retinal tears, the retinal break in Case 1 can be attributed to the pharmacologic action of ocriplasmin in inducing a posterior vitreous detachment rather than the physical action of an intravitreal injection in yielding vitreoretinal traction. This is supported by the fact that no retinal breaks were noted on dilated fundus examination 1 week after the intravitreal injection and the patient's relevant symptoms began 12 days after the injection. In both cases, ocriplasmin's pharmacologic action, while relieving the patients' vitreomacular traction, resulted in an incomplete or 'anomalous' PVD: the extent of ocriplasmin-mediated vitreous liquefaction not only released macular vitreoretinal adhesion, but also caused vitreoschisis that left residual vitreous cortex attached to the retina which ultimately contributed to a retinal break. ${ }^{2}$ Our observation diverges from preclinical studies, which note that in addition 
to inducing vitreous liquefaction, ocriplasmin produces complete separation of the vitreous cortex from the internal limiting membrane. ${ }^{10}$ This has, in fact, previously been cited as one of the advantages of ocriplasmin's use over surgical PVD induction, which was deemed less favorable in yielding incomplete cortical removal from the retinal surface with the consequent potential for fibrocellular proliferation and vitreoretinal traction. ${ }^{11,12}$ Anomalous PVD formation has, however, previously been implied in ocriplasmin-treated eyes which eventually underwent vitrectomy as surgeons reportedly encountered intraoperative vitreoschisis and continued vitreoretinal adhesion in these eyes. ${ }^{2}$ While it is not surprising that an anomalous PVD occurs in ocriplasmin treated eyes which suffer treatment failure, our cases are notable in that the vitreomacular traction was successfully relieved, yet both patients still suffered an anomalous PVD, and consequently, retinal breaks.

One harbinger of this case series is perhaps Phase III clinical trials of ocriplasmin where $26.5 \%$ of ocriplasmin-injected eyes were observed to have resolution of vitreomacular adhesion, yet only $13.4 \%$ of those eyes demonstrated a total PVD by ultrasonography. ${ }^{6}$ In the same vein, it is perhaps unfeasible for early pharmacologic generation of an anomalous PVD to be ameliorated non-surgically more than 24 hours after intravitreal injection, as the second order (autoproteolytic) kinetics describing ocriplasmin's concentration result in its decrease by over $97 \%$ during this interval. ${ }^{13,14}$ Thus, in patients treated with ocriplasmin, maintained vigilance for retinal breaks even after resolution of vitreomacular traction is critical with immediate evaluation of all symptomatic patients.

\section{Disclosure}

$\mathrm{T}$ Leng has served as a paid consultant to Thrombogenics, Inc. The other authors have no financial or proprietary interest in a product, method, or material published in this paper.

\section{References}

1. Krebs I, Brannath W, Glittenberg C, Zeiler F, Sebag J, Binder S. Posterior vitreomacular adhesion: a potential risk factor for exudative age-related macular degeneration? Am J Ophthalmol. 2007;144(5):741-746.

2. Rhéaume MA, Vavvas D. Pharmacologic vitreolysis. Semin Ophthalmol. 2010;25(5-6):295-302.

3. Robison CD, Krebs I, Binder S, et al. Vitreomacular adhesion in active and end-stage age-related macular degeneration. Am J Ophthalmol. 2009;148(1):79-82.e2.

4. Jerdan JA, Kao L, Glaser BM. The inner limiting membrane: a modified basement membrane? Invest Ophthalmol Vis Sci. 1986; 27(Suppl):230a.

5. Chen W, Mo W, Sun K, Huang X, Zhang YL, Song HY. Microplasmin degrades fibronectin and laminin at vitreoretinal interface and outer retina during enzymatic vitrectomy. Curr Eye Res. 2009;34(12): 1057-1064.

6. Stalmans P, Benz MS, Gandorfer A, et al. Enzymatic vitreolysis with ocriplasmin for vitreomacular traction and macular holes. $N$ Engl $J$ Med. 2012;367(7):606-615.

7. Kim BT, Schwartz SG, Smiddy WE, et al. Initial outcomes following intravitreal ocriplasmin for treatment of symptomatic vitreomacular adhesion. Ophthalmic Surg Lasers Imaging Retina. 2013;44: 334-343.

8. de Smet MD, Gandorfer A, Stalmans P, et al. Microplasmin intravitreal administration in patients with vitreomacular traction scheduled for vitrectomy: the MIVI I trial. Ophthalmology. 2009;116(7):1349-1355, 1355.e1-e2.

9. Benz MS, Packo KH, Gonzalez V, et al. A placebo-controlled trial of microplasmin intravitreous injection to facilitate posterior vitreous detachment before vitrectomy. Ophthalmology. 2010;117:791-797.

10. Gandorfer A, Rohleder M, Sethi C, et al. Posterior vitreous detachment induced by microplasmin. Invest Ophthalmol Vis Sci. 2004;45: 641-647.

11. Gandorfer A. Pharmacologic vitreolysis: rationale, potential indications, and promising agents. Retina. 2012;32(Suppl 2):S221-S224.

12. Johnson MW. How should we release vitreomacular traction: surgically, pharmacologically, or pneumatically? Am J Ophthalmol. 2013;155: 203-205.e1.

13. de Smet MD, Jonckx B, Vanhove M, van Calster J, Stalmans P, Stassen JM. Pharmacokinetics of ocriplasmin in vitreous. Invest Ophthalmol Vis Sci. 2012;53:8208-8213.

14. ThromboGenics. JETREA (ocriplasmin): US prescribing information http://www.accessdata.fda.gov/drugsatfda_docs/labe1/2012/ 125422s000lbl.pdf. Accessed May 1, 2014.
Clinical Ophthalmology

\section{Publish your work in this journal}

Clinical Ophthalmology is an international, peer-reviewed journal covering all subspecialties within ophthalmology. Key topics include: Optometry; Visual science; Pharmacology and drug therapy in eye diseases; Basic Sciences; Primary and Secondary eye care; Patient Safety and Quality of Care Improvements. This journal is indexed on Submit your manuscript here: http://www.dovepress.com/clinical-ophthalmology-journal

\section{Dovepress}

PubMed Central and CAS, and is the official journal of The Society of Clinical Ophthalmology (SCO). The manuscript management system is completely online and includes a very quick and fair peer-review system, which is all easy to use. Visit http://www.dovepress.com/ testimonials.php to read real quotes from published authors. 